\title{
Pyrotinib for metastatic breast cancer after multi-anti-HER2 targeted therapy: a case report
}

\author{
Feng $\mathrm{Li}^{1,2}, \mathrm{Li} \operatorname{Bian}^{1}$, Zerui $\mathrm{Qu}{ }^{1}$, Jianbin $\mathrm{Li}^{1,3}$, Fengrui Xu ${ }^{1,2}$, Zefei Jiang ${ }^{1}$ \\ ${ }^{1}$ Department of Breast Oncology, The Fifth Medical Center of Chinese PLA General Hospital, Beijing 100071, China; ${ }^{2}$ Department of Breast \\ Oncology, Academy of Military Medical Sciences, Beijing 100089, China; ${ }^{3}$ Medical Molecular Biology, Beijing Institute of Biotechnology, Beijing \\ 100071, China \\ Correspondence to: Li Bian. Department of Breast Oncology, The Fifth Medical Center of Chinese PLA General Hospital, No. 8 East Street, Fengtai \\ District, Beijing 100071, China. Email: bianli2013@aliyun.com.
}

\begin{abstract}
We report a typical case of human epidermal growth factor receptor-2 (HER2)-positive breast cancer with multiple metastases after multi-anti-HER2 therapy which responded to afterwards pyrotinib and gained significantly longer progression free survival compared with other cases. A 41 years-old woman was diagnosed with HER2-positive breast cancer in 2011 and developed liver, lung and bone metastases in 2014 after neoadjuvant therapy, radical mastectomy, adjuvant chemotherapy and targeted therapy. She then received multi-anti-HER2 therapy with trastuzumab, lapatinib and T-DM 1. The patient later got brain metastases and received local resection. In the end, the patient received pyrotinib $400 \mathrm{mg}$. She then reached partial response and lung metastases were significantly improved. The patient was then followed-up and achieved 29 months progression free survival. We also have applied next generation sequencing (NGS) to demonstrate the efficacy of anti-HER2 treatment. This case provides a reference for future treatment of refractory breast cancer.
\end{abstract}

Keywords: Metastatic breast cancer; anti-HER2 targeted therapy; pyrotinib; next generation sequencing (NGS); ctDNA; case report.

Received: 28 February 2020; Accepted: 24 March 2020; Published: 10 April 2020.

doi: $10.21037 /$ tbcr.2020.04.02

View this article at: http://dx.doi.org/10.21037/tbcr.2020.04.02

\section{Introduction}

Human epidermal growth factor receptor-2 (HER2)positive breast cancer has a higher rate of recurrence and poor prognosis (1). Patient life expectancy can be greatly be prolonged by anti-HER2 targeted drugs. Both the NCCN and Chinese Society of Clinical Oncology Breast Cancer (CSCO BC) guidelines recommend trastuzumab as the firstline anti-HER2 targeted drug for HER2-positive metastatic breast cancer. In the case of trastuzumab treatment failure, T-DM1 or lapatinib combined with capecitabine can be used as the next line of treatment. Unfortunately, some patients will eventually become resistant despite the clinical benefits achieved, and palliative care is generally recommended in the guidelines for multi-anti-HER2 targeted therapy treatment failure.

The development of new drugs may generate new prospects for these patients. Pyrotinib, an oral, irreversible pan-ErbB receptor tyrosine kinase inhibitor with activity against HER1, HER2, and HER4 (2), approved for HER2positive, relapsed or metastatic breast cancer that has received trastuzumab. Here, we report a typical case of HER2-positive breast cancer with brain and lung metastases who achieved long progression free survival (29 months) from pyrotinib after the multi-anti-HER2 treatments, and this case is written in accordance with CARE GUIDELINE.

\section{Case presentation}

This patient was diagnosed with hormone receptornegative, HER2-positive breast cancer at the age of 41 in January 2011. Neoadjuvant therapy was used 4 cycles 


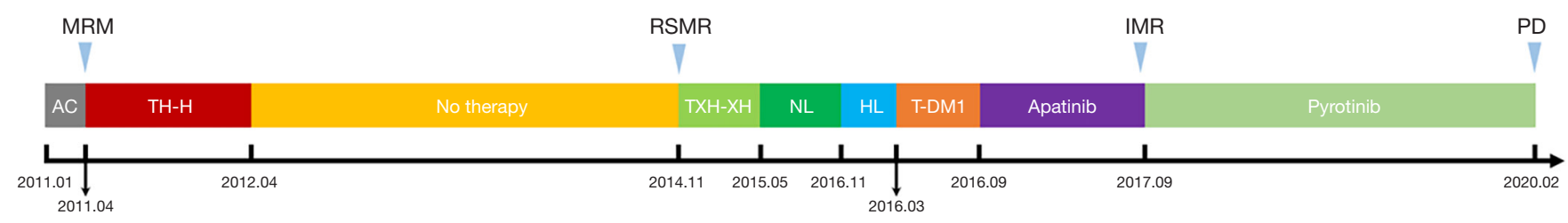

Figure 1 Timeline of the breast cancer patient's treatments. AC, doxorubicin hydrochloride liposome and cyclophosphamide; T, taxanes; H, trastuzumab; X, capecitabine; N, vinorelbine; L, lapatinib. T-DM1, trastuzumab emtansine; MRM, modified radical mastectomy, RSMR, right subclavian mass resection; IMR, brain metastases resection. $\mathrm{PD}$, progressive disease.
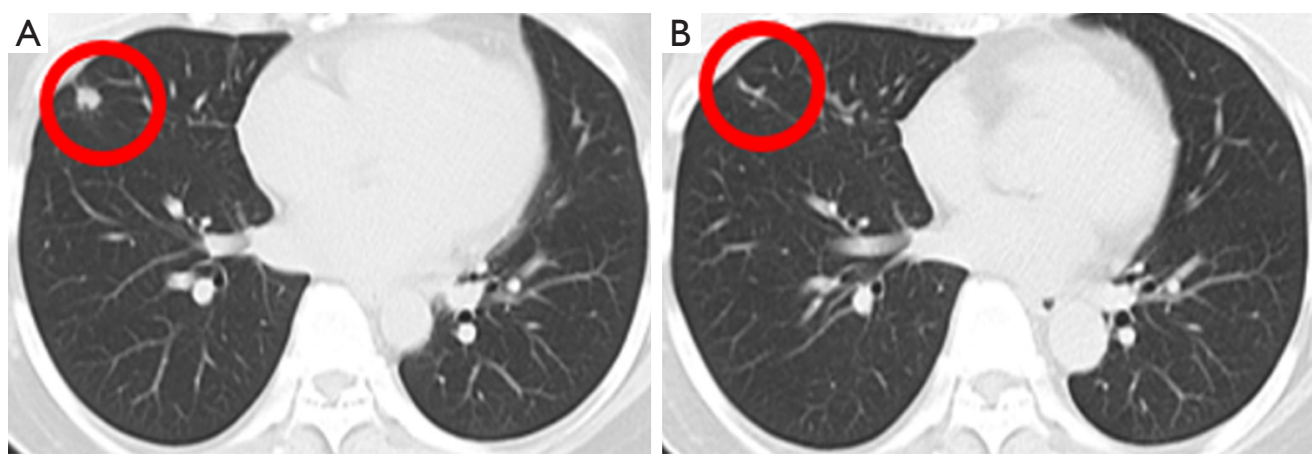

Figure 2 CT detection showing metastases before (A) and after treatment with pyrotinib (B: 2 cycles later).

and the protocol was doxorubicin hydrochloride liposome $\left(30 \mathrm{mg}, 18 \mathrm{mg} / \mathrm{m}^{2}, \mathrm{~d} 1\right)$ and cyclophosphamide $(1,000 \mathrm{mg}$, $\left.606 \mathrm{mg} / \mathrm{m}^{2}, \mathrm{~d} 1\right)$ every 3 weeks. After undergoing modified radical mastectomy of right breast, the pathological stage was $\mathrm{T} 2 \mathrm{~N} 1 \mathrm{M} 0$, and then she received adjuvant therapy with albumin-bound paclitaxel (400 mg, $242 \mathrm{mg} / \mathrm{m}^{2}, \mathrm{~d} 1$ ) in combination with trastuzumab $(8 \mathrm{mg} / \mathrm{kg}$, then $6 \mathrm{mg} / \mathrm{kg}$ ) every 3 weeks for 4 cycles and trastuzumab $(6 \mathrm{mg} / \mathrm{kg}$, week 3) until a year. In November 2014, she developed liver, lung, bone, and subclavian lymph node metastases, the pathological and immunohistochemical results of the subclavian lymph node metastases are the same as those of the primary breast, the disease free survival was 41 months. Therefore, combination therapy comprising trastuzumab $(6 \mathrm{mg} / \mathrm{kg}, \mathrm{d} 1)$, capecitabine $(3,000 \mathrm{mg}$, $\left.1,818 \mathrm{mg} / \mathrm{m}^{2}, \mathrm{~d} 1\right)$ and docetaxel $\left(120 \mathrm{mg}, 73 \mathrm{mg} / \mathrm{m}^{2}, \mathrm{~d} 1\right)$ every 3 weeks was initiated, which is the recommended first-line therapy for HER2-positive metastases breast cancer by CSCO BC guideline. After 6 cycles, the combined chemotherapy was changed to trastuzumab and capecitabine to avoid damage caused by docetaxel, the assessment result of the treatment was partial response (PR) according RECIST v1.1, the PFS was
6 months. The patient further received regular vinorelbine (40 mg, $\left.24 \mathrm{mg} / \mathrm{m}^{2}, \mathrm{~d} 1, \mathrm{~d} 8\right)$ and lapatinib (1,000 mg, d1) every 3 weeks for 8 cycles and achieved SD, then the treatment program was stopped due to adverse reactions. Subsequently, she underwent multi-anti-HER2 treatments including trastuzumab in combination with lapatinib and achieved PR, the PFS was 4 months, T-DM1 $(3.6 \mathrm{mg} / \mathrm{kg}$, week 3) achieved PR, the PFS was 6 months. Then she received apatinib and achieved PR, the PFS was 12 months (Figure 1). After clinical benefits have been achieved for all anti-HER2 treatments drugs, the patient was found to have brain metastases and underwent resection of metastases lesions. Based on clinical experience and demand of the patient, we tried to give her pyrotinib (400 mg, qd) as single agent after brain metastases resection. Following two cycles of therapy, the lung metastases of this patient significantly reduced and reached PR with CT evaluation (Figure 2), and the whole evaluation also reached PR. The only toxicity (grade 3-4) the patient experienced was diarrhea. To February 2020, the patient's cervical lymph nodes were enlarged and intracranial metastases were found again, the progression-free survival was 29 months.

At the beginning of pyrotinib, we performed the next 


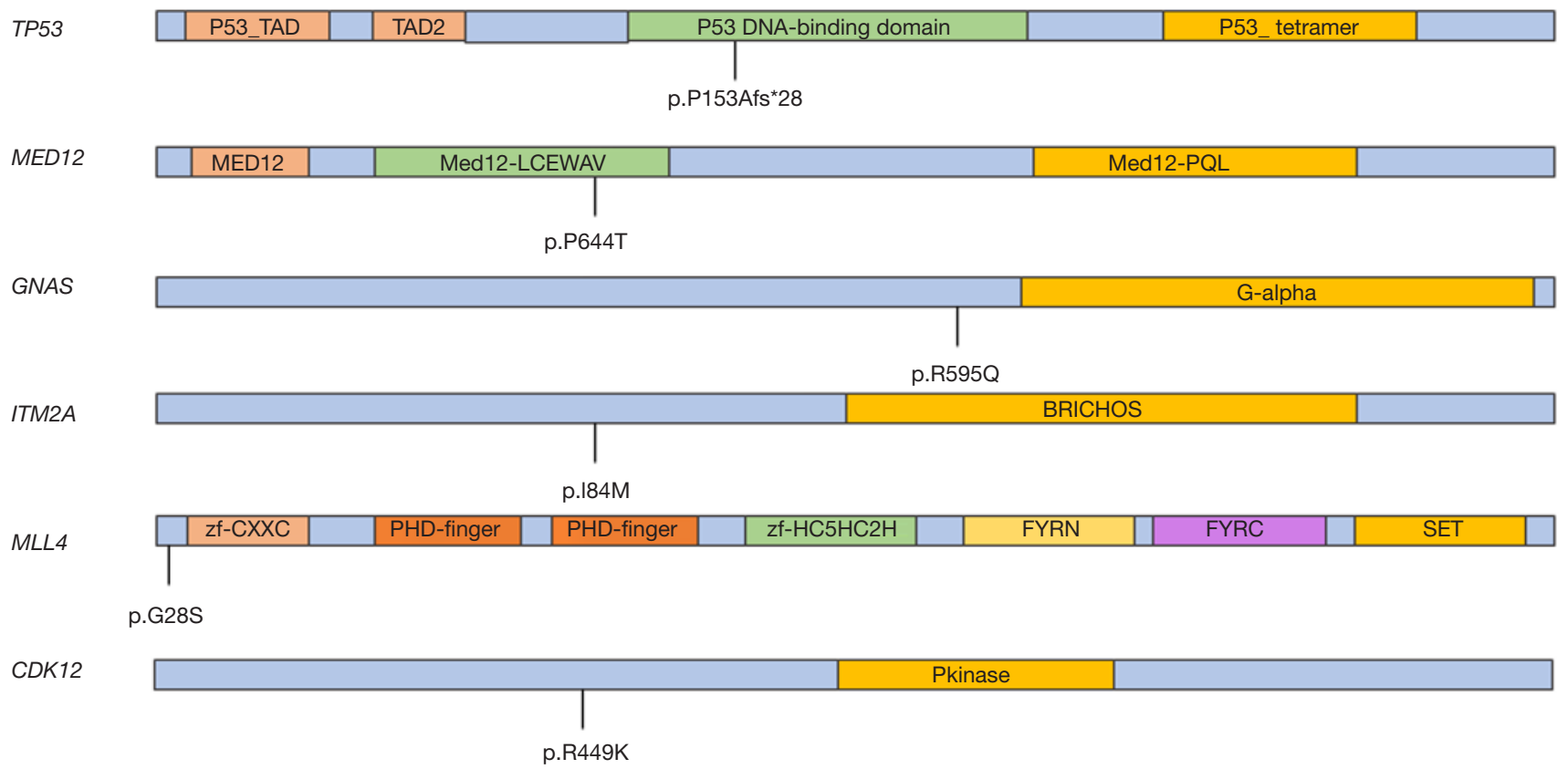

Figure 3 The p.P153Afs 28 mutation in TP53, the p.P644T mutation in MED12, the p.R595Q mutation in GNAS, the p.I84M mutation in ITM2A, the p.G28S mutation in MLL4 and p.R449K mutation in CDK12 genes identified by NGS. NGS, next generation sequencing.

generation sequencing (NGS) test for the patient to verify the validity of our treatment recommendation after communicating with the patient and her family members. We detected the expressions of 1,021 tumor-related genes in the NGS test of circulating tumor DNA (ctDNA). The result revealed 10 somatic mutations, including 4 copy number variants ERBB2, CCNE1, MLL4, and CDK12 amplification and 6 point mutations TP53 p.P153Afs*28, MED12 p.P644T, GNAS p.R595Q, ITM2A p.184M, MLL4 p.G28S and CDK12 p.R499K (Figure 3). The ERBB2 and CCNE1 amplification means that this patient is sensitive to anti-HER2 treatment.

\section{Discussion}

Targeted therapy for HER2-positive breast cancers is widely applied in current clinical practices (3). However, clinicians often face difficulties in giving treatment recommendations for patients after failure of multi-antiHER2 therapy. This patient got clinical benefit during the treatments of trastuzumab, lapatinib, and T-DM1, indicating that anti-HER2 treatment is effective for her. Previous study (4) has showed the outcome of patients with HER2-positive metastatic breast cancer can be significantly improved by continuous anti-HER 2 treatments. Therefore, as a new and accessible anti-HER2 targeted drug, pyrotinib was selected as subsequent treatment. In previous clinical studies, pyrotinib showed remarkable systemic activity in patients with HER2 positive metastatic breast cancer $(2,5)$, and PFS was 5.5 months in the pyrotinib monotherapy group in PHENIX study (6). Surprisingly, pyrotinib achieved long-term benefits for this patient. To the best of our knowledge, this is the typical case of brain and lung metastases from breast cancer that responded to pyrotinib after multi-anti-HER2 therapy, the PFS has lasted 29 months, which was significantly longer than previous study.

The patient develops a new intracranial metastasis before receiving pyrotinib. Studies shows that HER2 status was a strong predictor of survival after brain metastasis, and survival time of HER2-positive patients is significantly longer than HER2-negative patients (18 vs. 11 months, $\mathrm{P}=0.02)(7)$. Although the intracranial lesion has been surgically removed, drugs are still needed for systemic treatment. Pyrotinib is believed to penetrate the bloodbrain barrier more easily owing to its small molecular size, and it also shows significant systemic activity in patients with brain metastases from HER2-positive advanced breast 
cancer (6).

In the era of precision medicine, new diagnostic and therapeutic technologies can help clinicians to perform more appropriate treatments. NGS is crucial for the optimal cancer treatment (8). We received ctDNA of this patient from plasma and brain metastases to find potential targeted therapeutic opportunities. We observed that the brain tissue ctDNA has a significantly higher sensitivity than plasma, which may be due to tumor heterogeneity, and we also captured rarely documented somatic mutations in the genes CDK12 p.R499K in plasma (9). In brain tissue, there are 5 -point mutations and 4 copy number variations amplification. All point mutations except TP53 were not recorded and their clinical significance was unknown in the MSK database. Meanwhile, ERBB2 amplification indicates that the patient is sensitive to anti-HER2 drugs. However, CCNE1 amplification is a mechanism of trastuzumab resistance in HER2 positive breast cancer (10). For this reason, pyrotinib, another anti-HER2 drug that is different in mechanism from trastuzumab will remain an option for further treatment.

The strength of this article is showed the good efficacy and tolerability of pyrotinib. But there are some restrictions. We still need data from phase III clinical trials to compare the efficacy and safety of pyrotinib, and combine NGS to find beneficiaries who have received multi-anti-HER2 treatments.

\section{Conclusions}

Here we reported a metastatic breast cancer patient who's multi-anti-HER2 targeted therapy failed and was treated with pyrotinib instead. Through this case, we've discovered that patients who have received multi-anti-HER2 targeted therapies, doctors' clinical experience and NGS can provide new options for subsequent treatment if treatment guidelines fail to provide recommendations.

\section{Acknowledgments}

Funding: This study was supported by The National Natural Science Foundation of China (Grant number 81602314).

\section{Footnote}

Guideline Checklist: The authors have completed the CARE checklist (available at http://dx.doi.org/10.21037/ tbcr.2020.04.02).
Conflicts of Interest: All authors have completed the ICMJE uniform disclosure form (available at http://dx.doi. org/10.21037/tbcr.2020.04.02). ZJ serves as an unpaid Editor-in-Chief of Translational Breast Cancer Research. JL serves as an unpaid Managing Editor of Translational Breast Cancer Research. The other authors have no conflicts of interest to declare.

Ethical Statement: The authors are accountable for all aspects of the work including full data access, integrity of the data and the accuracy of the data analysis. The patient has given the consent for the case report to be published.

Open Access Statement: This is an Open Access article distributed in accordance with the Creative Commons Attribution-NonCommercial-NoDerivs 4.0 International License (CC BY-NC-ND 4.0), which permits the noncommercial replication and distribution of the article with the strict proviso that no changes or edits are made and the original work is properly cited (including links to both the formal publication through the relevant DOI and the license). See: https://creativecommons.org/licenses/by-nc-nd/4.0/.

\section{References}

1. Slamon DJ, Godolphin W, Jones LA, et al. Studies of the HER-2/neu proto-oncogene in human breast and ovarian cancer. Science 1989;244:707-12.

2. Ma F, Li Q, Chen S, et al. Phase I Study and Biomarker Analysis of Pyrotinib, a Novel Irreversible Pan-ErbB Receptor Tyrosine Kinase Inhibitor, in Patients With Human Epidermal Growth Factor Receptor 2-Positive Metastatic Breast Cancer. J Clin Oncol 2017;35:3105-12.

3. Harbeck N, Gnant M. Breast cancer. Lancet 2017;389:1134-50.

4. Krop IE, Kim SB, Martin AG, et al. Trastuzumab emtansine versus treatment of physician's choice in patients with previously treated HER2-positive metastatic breast cancer (TH3RESA): final overall survival results from a randomised open-label phase 3 trial. Lancet Oncol 2017;18:743-54.

5. Ma F, Ouyang Q, Li W, et al. Pyrotinib or Lapatinib Combined With Capecitabine in HER2-Positive Metastatic Breast Cancer With Prior Taxanes, Anthracyclines, and/or Trastuzumab: A Randomized, Phase II Study. J Clin Oncol 2019;37:2610-9.

6. Jiang Z, Yan M, Hu X, et al. Pyrotinib combined with capecitabine in women with HER2 + metastatic breast 
cancer previously treated with trastuzumab and taxane. J Clin Oncol 2019;37:abstr 1001.

7. Shen Q, Sahin AA, Hess KR, et al. Breast cancer with brain metastases: clinicopathologic features, survival, and paired biomarker analysis. Oncologist 2015;20:466-73.

8. Dawson SJ, Tsui DW, Murtaza M, et al. Analysis of circulating tumor DNA to monitor metastatic breast cancer. N Engl J Med 2013;368:1199-209.

doi: $10.21037 /$ tbcr.2020.04.02

Cite this article as: Li F, Bian L, Qu Z, Li J, Xu F, Jiang Z. Pyrotinib for metastatic breast cancer after multi-anti-HER2 targeted therapy: a case report. Transl Breast Cancer Res 2020;1:8.
9. Joshi PM, Sutor SL, Huntoon CJ, et al. Ovarian cancerassociated mutations disable catalytic activity of CDK12, a kinase that promotes homologous recombination repair and resistance to cisplatin and poly(ADP-ribose) polymerase inhibitors. J Biol Chem 2014;289:9247-53.

10. Scaltriti M, Eichhorn PJ, Cortés J, et al. Cyclin $\mathrm{E}$ amplification/overexpression is a mechanism of trastuzumab resistance in HER2+ breast cancer patients. Proc Natl Acad Sci U S A 2011;108:3761-6. 